\title{
High-Resolution MRI of Intracranial Atherosclerotic Disease
}

\author{
Chang-Woo Ryu, MD', Hyo-Sung Kwak, MD², Geon-Ho Jahng, PhD', Han Na Lee, MD'
}

Intracranial atherosclerotic disease (ICAD) causes up to $10 \%$ of all ischemic strokes, and the rate of recurrent vascular ischemic events is very high. Important predictors of vulnerability in atherosclerotic plaques include the degree of stenosis and the underlying plaque morphology. Vascular wall MRI can provide information about wall structures and atherosclerotic plaque components. High-resolution (HR)MRI in ICAD poses a greater challenge in the neurologic fields, because a high in-plane resolution and a high signal-to-noise ratio are required for vessel wall imaging of ICAD. Until now, plaque imaging of ICAD has focused on assessing the presence of a plaque and evaluating the plaque load. Going forward, evaluation of plaque vulnerability through analysis of imaging characteristics will be a critical area of research. This review introduces the acquisition protocol for HR-MRI in ICAD and the current issues associated with imaging.

Key Words : Intracranial atherosclerotic disease; Atherosclerosis; Cerebral arteries; Magnetic resonance imaging (MRI); Vessel wall imaging

Intracranial atherosclerotic disease (ICAD) is defined as atherosclerotic lesions affecting large intracranial arteries at the base of the brain, including the intracranial carotid artery (ICA), middle cerebral artery (MCA), and basilar artery, etc.

ICAD was once known as one of the most common subtype of strokes worldwide, especially in Asians

'Department of Radiology, Gangdong Kyung Hee University Hospital, College of Medicine, Kyung Hee University, Seoul, Korea; 2Department of Radiology, Jeonbuk National University Hospital, College of Medicine, Jeonbuk National University, Jeonju, Korea Received November 2, 2013; accepted after revision December 8, 2013.

Correspondence to: Chang-Woo Ryu, MD, Department of Radiology, Gangdong Kyung Hee University Hospital, 892 Dongnam-ro, Gangdong-gu, Seoul 134-727, Korea.

Tel. 82.2.440.6186 Fax. 82.2.440.6932

E-mail: md.cwryu@gmail.com

This is an Open Access article distributed under the terms of the Creative Commons Attribution Non-Commercial License (http://creativecommons.org/licenses/by-nc/3.0) which permits unrestricted non-commercial use, distribution, and reproduction in any medium, provided the original work is properly cited. and Hispanics [1-3]. However, ICAD has received less attention due to a focus on the more accessible extracranial carotid artery occlusive disease in the West. A study that showed a strikingly high prevalence of both intracranial plaque and intracranial stenosis in 339 autopsies of patient with stroke suggested that the real prevalence of ICAD in whites may be underestimated [4]. The increasing prevalence of risk factors for ICAD, such as diabetes and metabolic syndrome, also contribute to the increase in the prevalence of ICAD.

Only one or two decades ago, it was not easy to screen for ICAD in asymptomatic patients, because ICAD is not easily detected by physical examination or clinical history. However, with advances in $\mathrm{CT}$ and MR techniques making it easier to apply these modalities more widely, ICAD is being detected much more frequently. An increase in the diagnosis of ICAD has lead to an increase in new knowledge about stroke risk and the pathophysiology of ICAD.

High-resolution magnetic resonance imaging (HR- 
MRI) has been used recently to identify intracranial wall features [5, 6]. HR-MRI has several advantages compared to traditional MRI. In addition to visualization of the sub-millimeter arterial wall, it enables a high signal-to-noise ratio and minimal scan duration [5]. The degree of stenosis is no longer the sole predictor of vulnerable atherosclerotic lesions. Many investigations have revealed that various intraplaque factors, such as plaque morphology, plaque components, and inflammation, are also closely associated with the vulnerability of an atherosclerotic plaque. While conventional luminal images are limited to demonstrating the plaque, HR-MRI is able to directly demonstrate plaque characteristics.

In the field of neurointervention, characterization of an intracranial artery lesion is important for many reasons. The characteristics of an atherosclerotic lesion are known as major factors influencing the rate of periprocedural complications [7,8] and therefore can be used to select patients for percutaneous transluminal angioplasty and stenting, and for designing individualized operating plans [9].

Fortunately, HR-MRI of the intracranial artery is a good benchmarking model based on prior research involving carotid wall MRI. Based on numerous clinical studies of carotid endarterectomy, carotid wall MRI is emerging as the best candidate for assessing carotid stenosis with additional diagnostic features pertinent to patient management.

To date, this attention to ICAD and the creation of a benchmarking model has led to an increasing number of articles about HR-MRI for ICAD. In this article, on the basis of previous research and our experience, we describe the current protocol for intracranial HR-MRI and discuss and illustrate the role of HR-MRI, focusing on the vulnerability of ICAD, pathophysiology and differential diagnosis. In addition, we discuss current perspectives on the role of HR-MRI.

\section{HR-MRI PROTOCOL}

A major limitation of intracranial HR-MRI is that the intracranial arteries are too small to obtain adequate spatial resolution. Therefore, there are several requirements for HR-MRI of intracranial artery. First, examination of the intracranial arteries must be performed with high-field MRI (usually $3 \mathrm{~T}$ or $7 \mathrm{~T}$ in future investigations) to acquire a high signal-to-noise ratio. Next, the voxel size should be set to under $1 \times 1$ $\mathrm{mm}$. If needed, presaturation is added to the sequence for elimination of the signal within the vascular lumen.
Finally, we must consider that the intracranial arteries are surrounded by cerebrospinal fluid (CSF), unlike the carotid or coronary arteries. CSF flow can lead to a heterogeneous signal intensity around the vessel, and this can disturb the delineation of the outer margin of the intracranial artery. Fig. 1 is an example of HR-MRI of the MCA.

In the extracranial carotid artery, multimodal sequence is essential to characterize the components of an intraplaque lesion. T1-weighted MR and time-offlight MR angiography (TOF-MRA) are good at visualizing intraplaque hemorrhage. T2-weighted and proton density (PD) weighted sequences are suitable to differentiate between the lipid core and plaque cap. Enhancement can characterize inflammation or better visualize the margin of the plaque cap. The critical role of multimodal sequencing has been confirmed by many studies that compare pathology and MRI findings. The multimodality sequence can be acquired in intracranial HR-MRI, similar to carotid-wall MRI. However, with respect to the intracranial artery, there is no clear answer for whether multimodal MR sequences are helpful in analyzing the plaque component. It is quite difficult practically to harvest pathologic specimens from ICAD, which proves that the variables and change in signal intensity on multimodal sequencing can characterize the vulnerable lesions in ICAD. There has not been any investigation to correlate HR-MRI and pathologic specimens in ICAD.

If you are looking to try multimodal sequencing in order to provide multispectral characterization of an arterial wall plaque in a manner similar to that validated by carotid MR imaging, we recommend the HR-MRI protocol, including two-dimensional (2D) PD-, T1-, and T2-weighted turbo spin echo (TSE) sequences (Fig. 1). In our experience, PD-weighted imaging is best for visualizing the vessel margins and lumen. T1-weighted imaging is known to be a good technique for revealing intra-plaque hemorrhage, whereas the outer boundary is less clear. T2-weighted imaging depicts the inner boundaries with less clarity, but T2-weighted imaging can show a hyperintense band, which is thought to be the fibrotic cap around the lumen.

A 3D imaging technique with isotropic voxels is thought to improve the ability to detect subtle vessel wall lesions by minimizing partial volume effects. 3D imaging is also better for suppression of CSF flow artifact and for free reconstruction of any plane. Most of all, 3D may be beneficial in screening large populations, because raw data acquired with the axial plane 


\section{MRI of Intracranial Atherosclerotic Disease}

can be easily reconstructed with any plane. However, one drawback to 3D imaging is that it is not comparable to 2D imaging in terms of spatial resolution.

To clarify the border between the vessel wall and vessel lumen, signal from the intravascular flow should be eliminated, similar to blood flow imaging. Complete suppression of signal from the vessel lumen, through the black blood technique, is usually preferred for vascular wall imaging in most cases. Inflowing blood that enters the slice of an intracranial HR-MRI is unsaturated and shows strong signal intensity. Therefore, a pre-saturation pulse should be added to the caudal slice from the skull base. In our experience, contrary to carotid wall MRI, a preparation with cardiac gating is not required to reduce motion artifact caused by arterial pulsation.

The proper plane of acquisition should be selected according to the purpose of the examination, because the slice number is constrained by acquisition time. Imaging perpendicular to the vessel axis is favorable for measuring the plaque burden and the lumen, for localizing the distribution of the plaque (eccentric or concentric), and for characterizing the components of the plaque. The imaging plane should be directed along the axis of the vessel of interest that has been visualized on the previously acquired MRA. The main limitation of using this slice direction is the length of time required for acquisition.
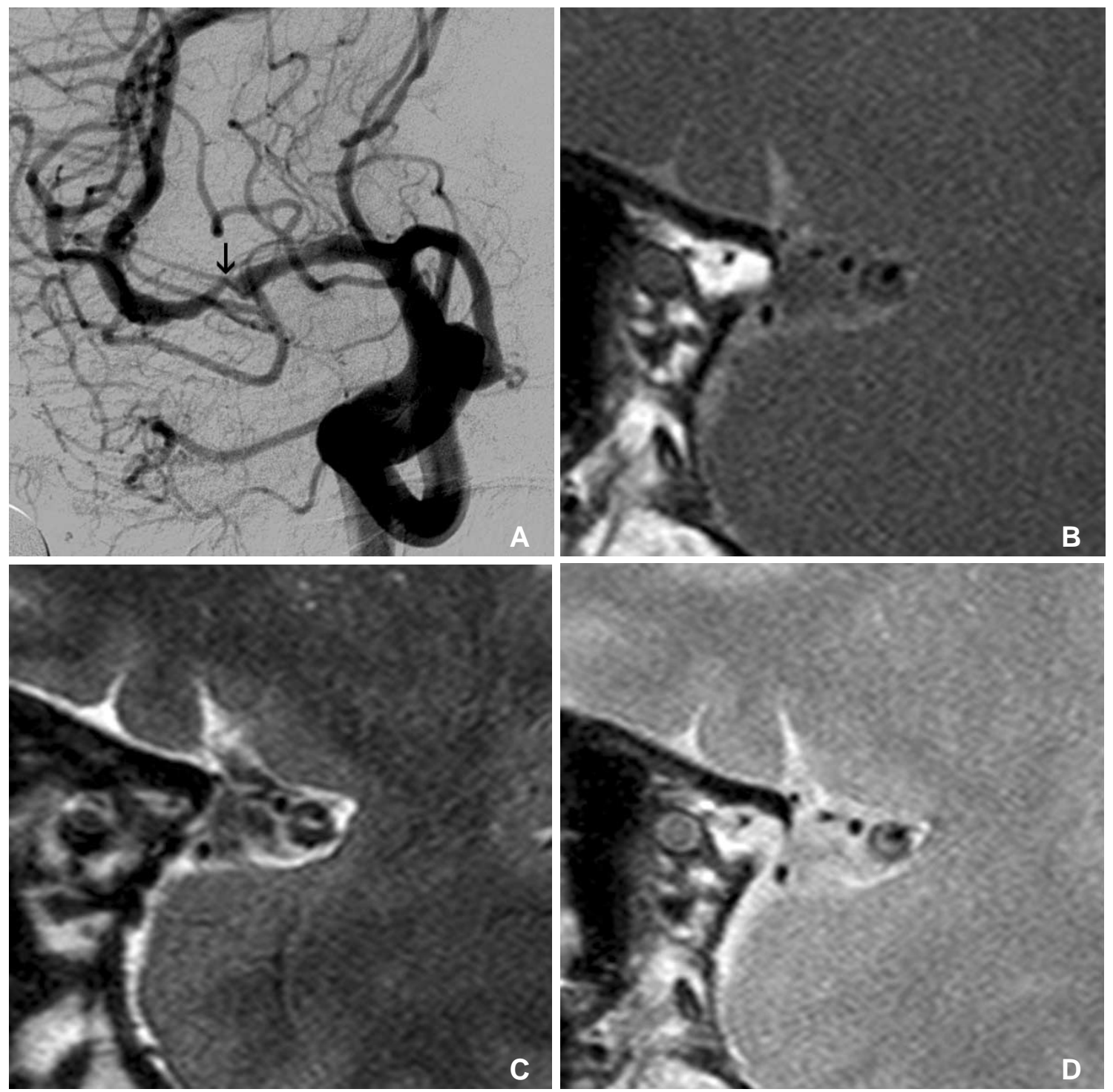

Fig. 1. Multimodal sequence can be acquired in intracranial HR-MRI, like as carotid wall MRI. This is an example of multimodal sequence of HR-MRI of MCA. Right carotid angiography shows severe stenosis at M1 (arrow on A). Figure B, C, and D are T1, T2 and proton density weighted HR-MRIs at this stenotic segment, consequently. These multimodal sequences of MRI show clearly thick and eccentric plaque and dark inner lumen at outer margin of MCA. 
Imaging parallel to the vessel axis is more favorable for screening for ICAD (Fig. 2). If you scan HR-MRI using a direction transverse to the head at the level of clinoid process, HR-MRI can show the whole vessel, including the Circle of Willis, distal ICAs, the whole length of M1, the anterior communicating artery and the basilar artery, without a long scan time. However, imaging parallel to the vessel axis is impossible if one wants to assess the plaque burden and distribution.

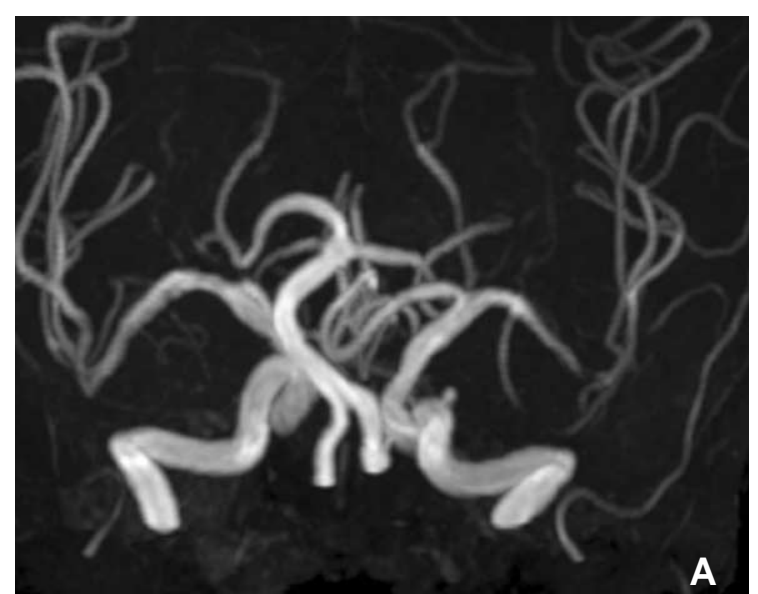

Fig. 2. Three dimensional T1-weighted FLAIR MRI reconstructed with axial plane can cover the wide field of view. A severe stenotic segment of left distal M1 on MR angiography (A) shows enhancing plaque (arrows) on axial contrast-enhanced T1 FLAIR MRI (B).

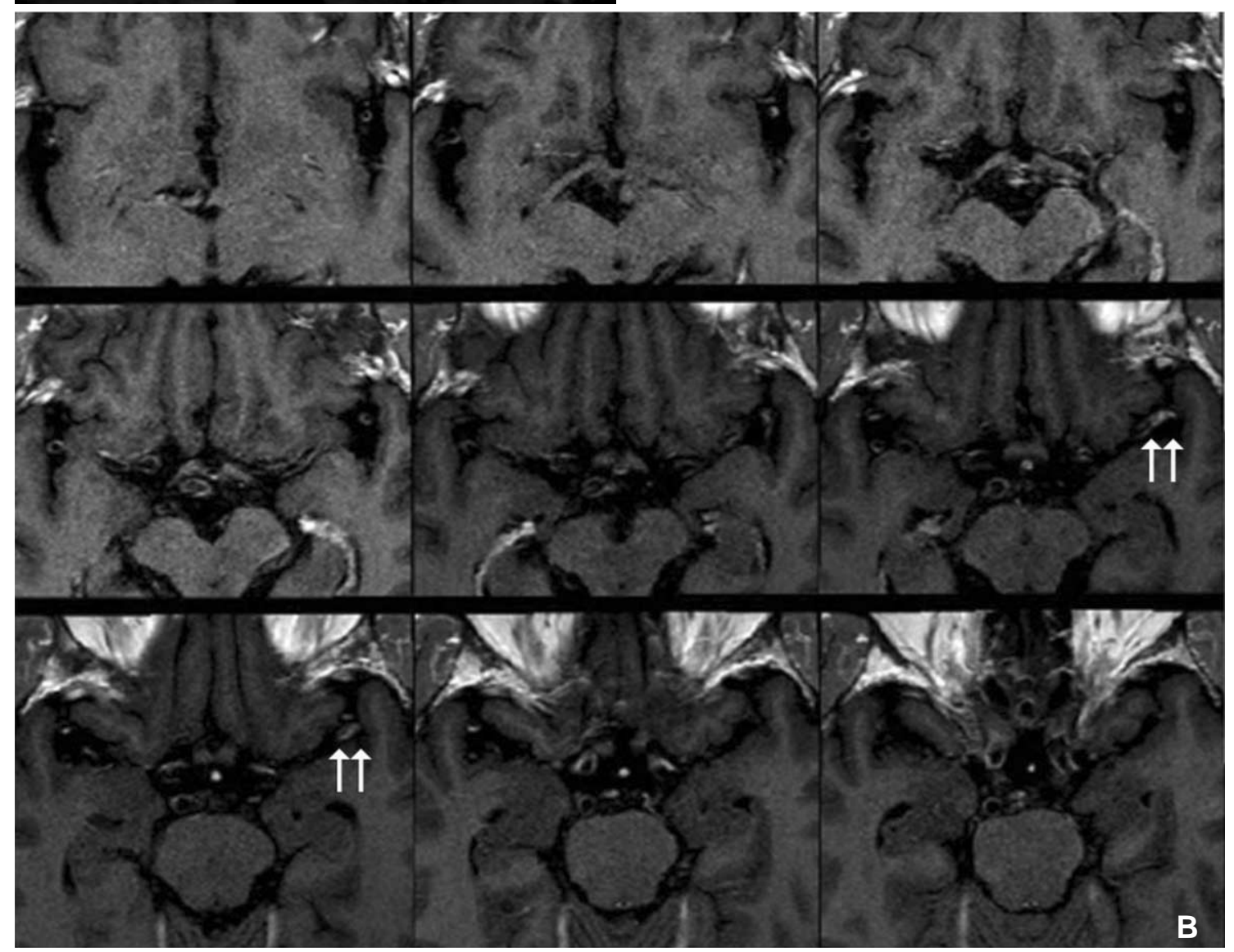


also focused on evaluating plaque vulnerability. Previously published investigations demonstrated that plaque vulnerability is related to the remodeling pattern, cross sectional area of the plaque burden, intraplaque hemorrhage and contrast enhancement on HRMRI.

\section{Remodeling pattern}

Atherosclerosis of vessels follows two different growth patterns: positive remodeling and negative remodeling. Positive remodeling is defined as a compensatory increase in vessel size in response to increasing plaque burden [12]. In contrast, negative remodeling is defined as local shrinkage of the vessel size $[13,14]$. Studies of the coronary arteries have shown that positive remodeling is significantly correlated with ischemic symptoms [15]. A possible explanation for correlation may be that positive remodeling in an area with more plaque has an increased risk of rupture, in contrast to an area of negative remodeling with fibrotic change that is more resistant to rupture. We found two articles that assessed the relationship between remodeling type and clinical symptoms of ischemic stroke $[5,16]$. In these studies, the symptomatic MCA stenosis group showed a higher prevalence of positive remodeling, whereas, the asymptomatic MCA stenosis group had more negative remodeling. Fig. 3 demonstrates examples of the positive and negative remodeling of MCA stenosis on HR-MRI.

\section{Plaque burden}

The volume of plaque has also been considered as a risk factor for stroke in the intracranial arteries $[5,6$, 16]. Two published articles demonstrated that symptomatic MCA stenosis had greater plaque thickness or a higher ratio of plaque thickness to patent lumen, compared to asymptomatic MCA stenosis. The area of plaque can offset the stenosis degree due to positive remodeling on conventional MRA and CTA. However, HR-MRI can provide information about the lumen status in nonstenotic atherosclerotic disease. Views perpendicular to the MCA axis should be obtained to clearly show the plaque and lumen areas.

In order for this novel diagnostic method to be a valuable clinical tool, it must be a reproducible qualitative and quantitative method. Two published studies revealed that the inter- and intra-observer agreements are excellent for the quantitative measurement of the plaque and lumen areas [9, 17].

One study directly comparing the degrees of stenosis detected by MRA and HR-MRI revealed that measurement of stenosis on HR-MRI correlates well with the degree of stenosis on MRA [12].

\section{Intraplaque hemorrhage}

Intraplaque hemorrhage (IPH) on carotid MRI is considered a risk factor for ischemic stroke [18]. In a recent meta-analysis of carotid plaque MRI in 779 subjects with a mean follow-up of greater than one month, the hazard ratio for IPH as predictors of subsequent stroke/transient ischemic attack was 4.59 (95\% confidence interval, 2.91-7.24) [19]. A study of
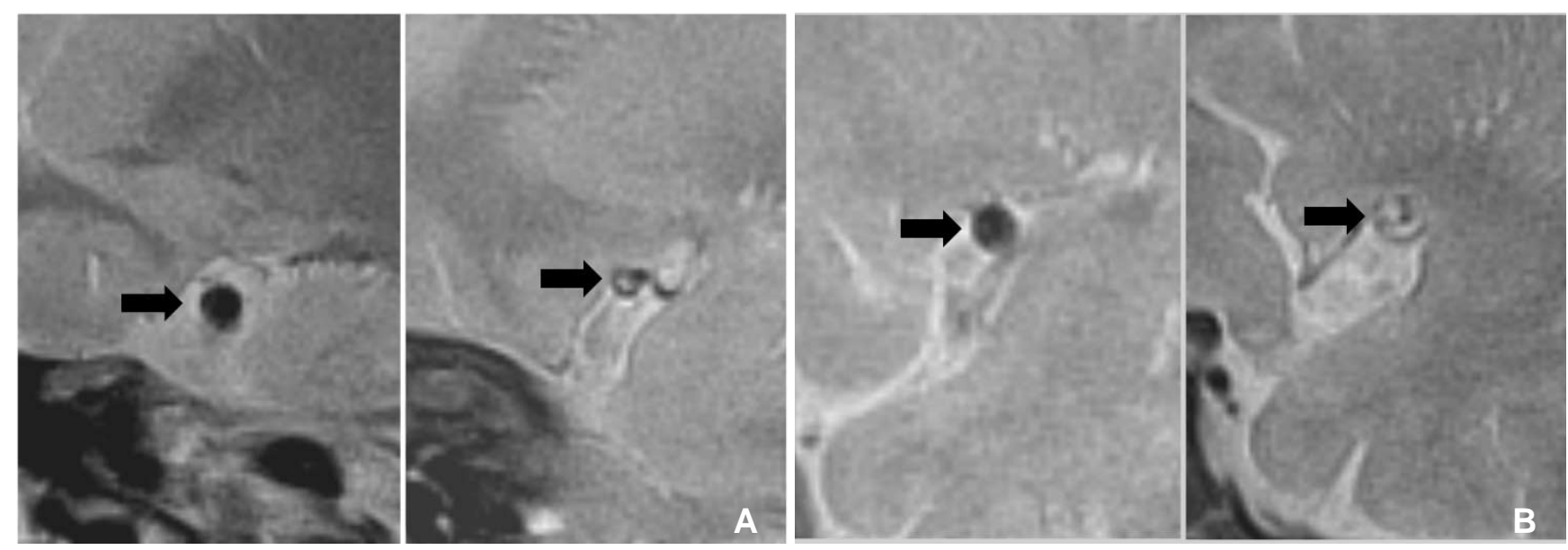

Fig. 3. These are examples of the remodeling of intracranial atherosclerotic disease. A. A 70-year-old man with asymptomatic MCA stenosis shows the smaller outer boundary of the stenotic segment (arrow on right-side figure) than contralateral side (arrow on left-side figure). B. Symptomatic MCA stenosis in a 67-year-old man shows the positive remodeling (arrow on right-side figure) which has the larger diameter than contralateral normal MCA (arrow on left-side figure). 
HR-MRI of the MCA also presented a different occurrence rate of IPH between symptomatic and asymptomatic MCAs [10].

Ryu et al. [6] reported that T1- and T2-hyperintense foci were more frequently observed within the plaques of symptomatic stenoses than within the plaques of asymptomatic stenoses. This study was the first to raise the possibility that a hyperintense lesion in plague may represent vulnerable lesion.

In a retrospective study of 109 high-grade (>70\%) MCA stenoses, recurrence of IPH was significantly different between symptomatic and asymptomatic MCAs (19.6\% vs. $3.2 \%$, p=0.01) [20]. However, one limitation of IPH detection through vulnerability is a low prevalence of IPH at the stenosis $(10.1 \%$ of 109 stenotic MCAs). Otherwise, two recent studies also presented that the prevalence of IPH in symptomatic stenosis could be higher than in asymptomatic patients $[17,21]$. We also experienced that IPH within plaque was closely related to vulnerability (Fig. 4). However, as stated above, the sensitivity of IPH was too low to apply to clinical screening. In HR-MRI, T1-weighted turbo spin echo imaging and magnetization-prepared rapid acquisition with gradient echo (MPRAGE) imaging are sensitive sequences for the detection of IPH (Fig. 5).

Whereas carotid wall MRI has various predictive imaging findings that reveal vulnerable histopathologic characteristics, other predictive findings besides IPH were not suggested on HR-MRI of ICAD. HR-MRI of ICAD has limited spatial resolution, making differentiation between the intraplaque lipid core and the fibrous cap difficult. A hyperintense band was observed in most of the MCA plaques, irrespective of symptoms,
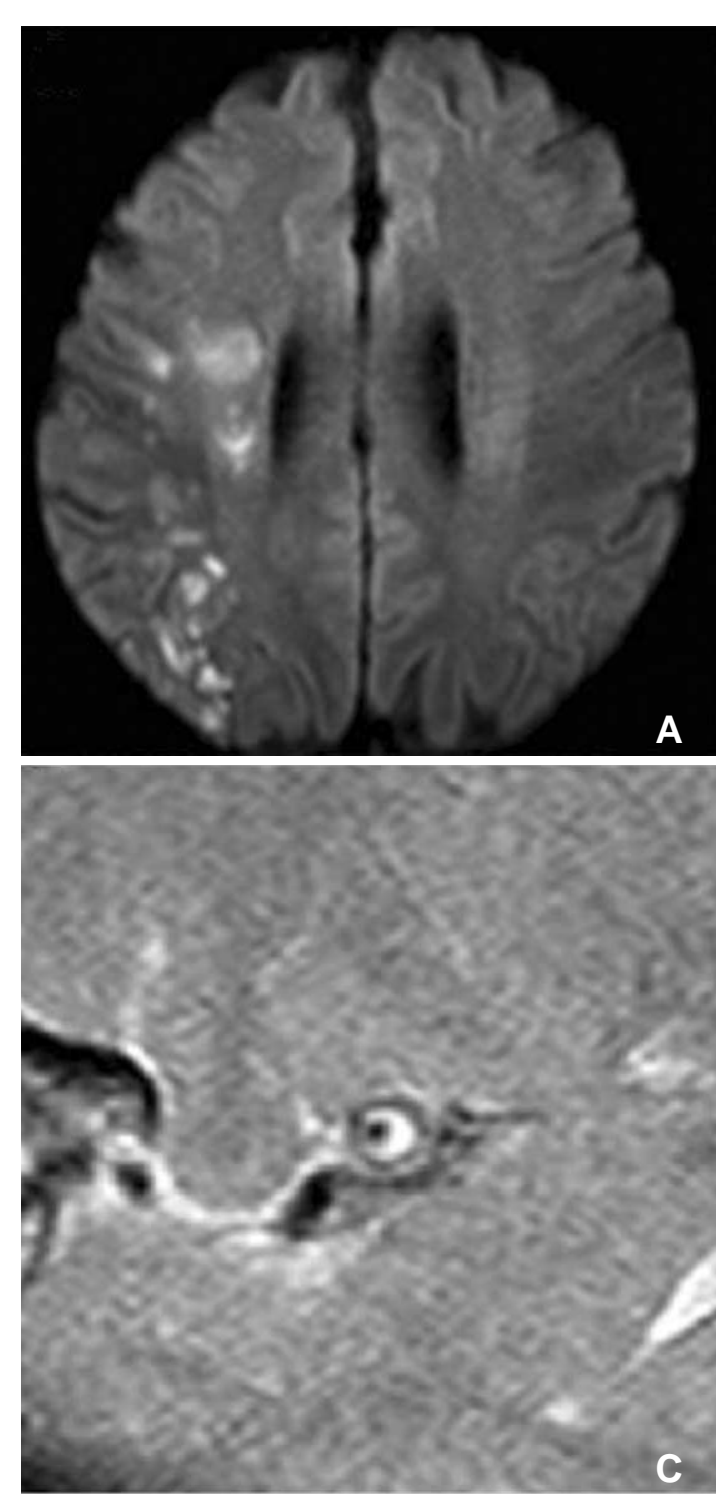

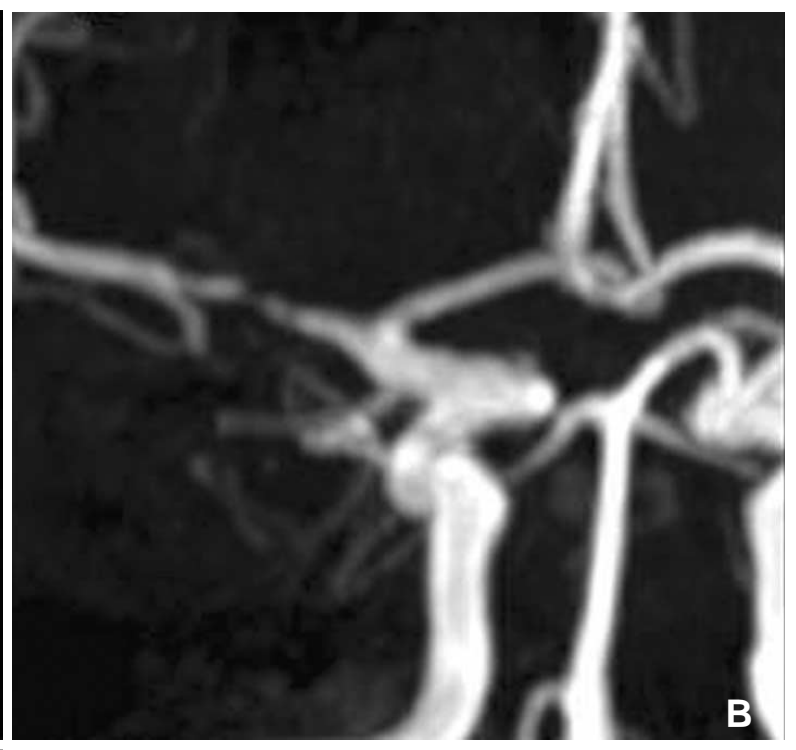

Fig. 4. Patients with multiple acute infarctions at right internal borderzone $(\mathbf{A})$ has severe stenosis at right $\mathrm{M} 1$ segment $(\mathbf{B})$. T1 weighted HR-MRI reveals that circumferential high signal intensity within plaque of stenotic segment $(\mathbf{C})$. 


\section{MRI of Intracranial Atherosclerotic Disease}

suggesting that this hyperintense band might represent a fibrotic component of the plaque, although at this point we have no direct evidence to support this assumption $[16,17]$.

\section{Contrast enhancement}

Strong contrast enhancement of the plaque is related to an increase in the vascular supply to the plaque and increased endothelial permeability that promotes entry of the contrast agent into the extravascular space [2225] (Fig. 6). Several recent investigations suggested that enhancement of the carotid plaque on carotid wall
MRI may be a predictable marker of clinical symptoms $[26,27]$. However, the evidence in support of this opinion is too weak to fully establish the role of assessment of plaque vulnerability in carotid imaging. The established role of contrast-enhanced sequences in multimodal MRI of the carotid is only to enhance demarcation of the fibrous cap from the carotid plaque.

Swartz et al. [28] were the first to report that enhancement of the plaque is seen only at the vessel supplying the area of acute infarction, even with multiple stenotic lesions. After this study, several studies regarding the relationship between enhancing
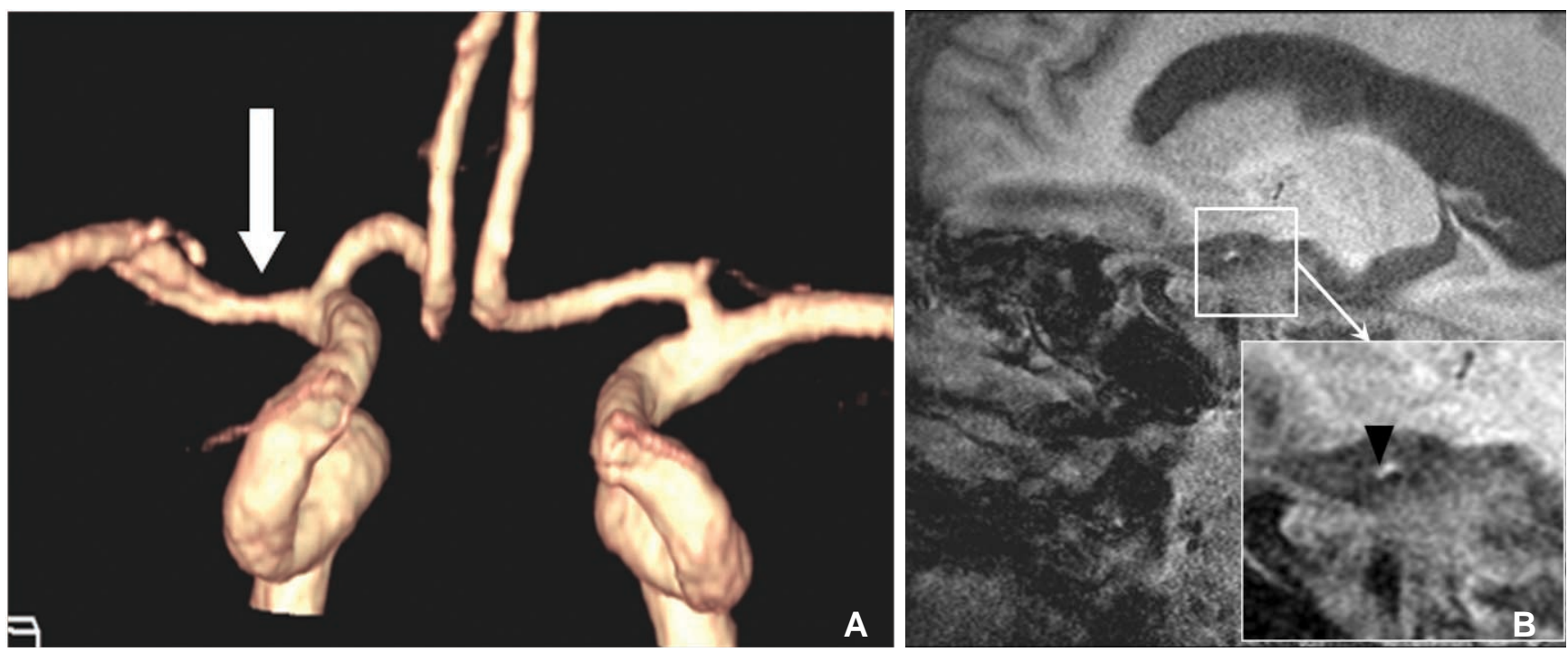

Fig. 5. MPRAGE MRI is one of the sensitive sequences for detection of intraplaque hemorrhage. A. MRA of symptomatic patient shows mild stenosis (arrow) at right M1. B. MPRAGE MRI shows hyperintense intraplaque hemorrhage (arrowhead) at this segment.
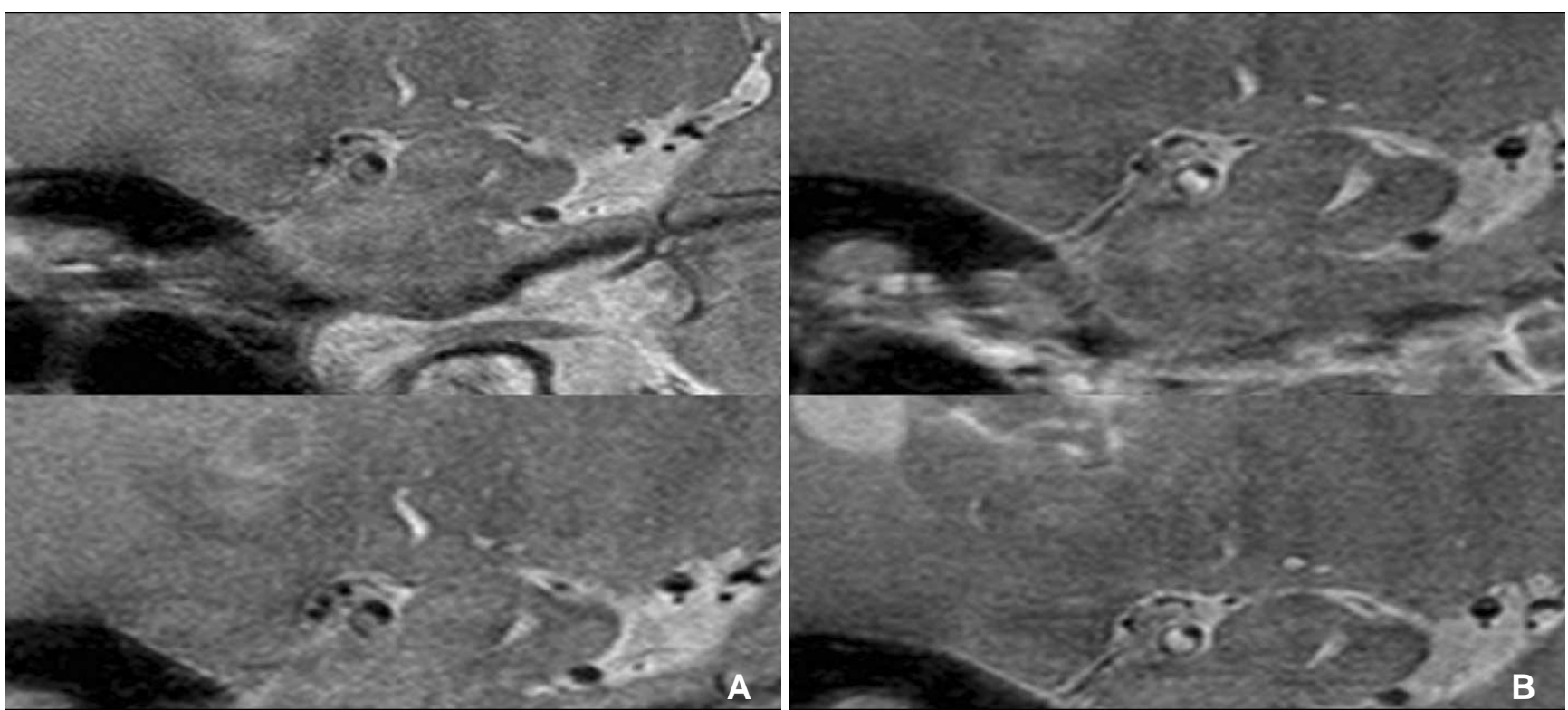

Fig. 6. An example of the enhancement of plaque at intracranial artery. A. Pre-contrast proton density weighted HR-MRI shows eccentric thick plaque at MCA. B. These plaques are strong enhanced on contrast-enhanced HR-MRI (Courtesy of DC Suh, MD). 
intracranial atherosclerotic plaques and acute ischemic stroke were published. Skarpathiotakis et al. [11] showed that strong pathologic enhancement of ICAD was seen in all patients imaged within four weeks of ischemic stroke in the vessel supplying the stroke territory. They also demonstrated a tendency towards decreasing enhancement as the time interval between imaging and ischemic events increased. Vakil et al. [10] also presented the results of a retrospective study that showed significant differences in contrast enhancement between the symptomatic and asymptomatic groups. In this retrospective study of 22 high-grade intracranial atherosclerotic disease plaques, plaque enhancement was observed in $70 \%$ of the symptomatic plaques and in $8 \%$ of the asymptomatic plaques [10].

However, there is some debate about whether contrast enhancement is a good prognostic tool for ischemic stroke for intracranial stenosis. Klein et al. [29] expressed doubt about the relationship between plaque
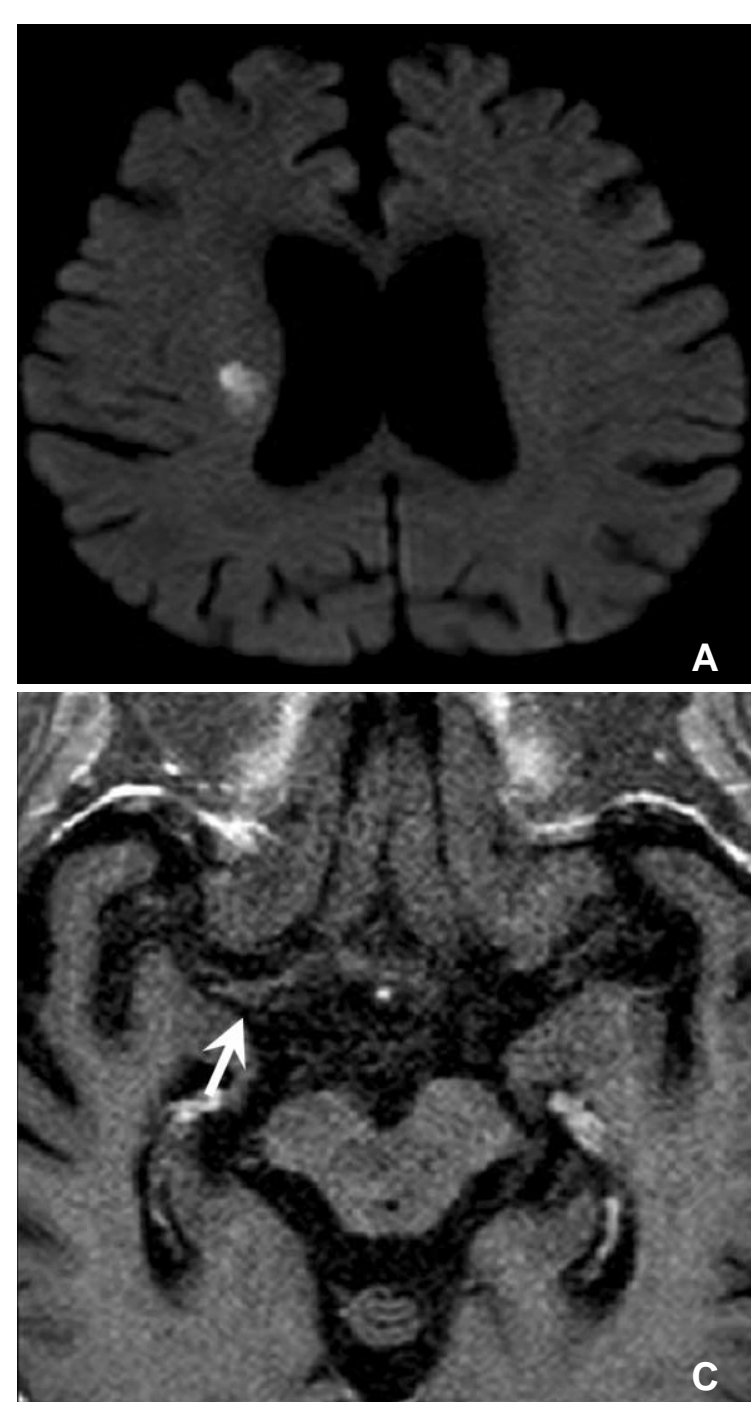

enhancement and stroke because they found stationary enhancement of the basilar plaque, regardless of the time elapsed since an acute event.

\section{KNOWLEDGE ABOUT PATHOPHYSIOLOGY OF PLAQUE AND INFARCTION}

\section{Plaque distribution}

Plaque distribution has been suggested as an important factor predictive of stroke related to atherosclerosis. According to a previous coronary artery study, plaque tends to form at positions opposite to flow dividers [11], and plaque at flow dividers is exposed to high shear stress, which is the mechanical trigger of plaque rupture. Two previous studies of ICAD produced similar results to coronary atherosclerosis $[30,31]$. In these studies, plaque was dominantly located at the ventral wall of the MCA and basilar arteries, which are opposite the orifice of the perforat-

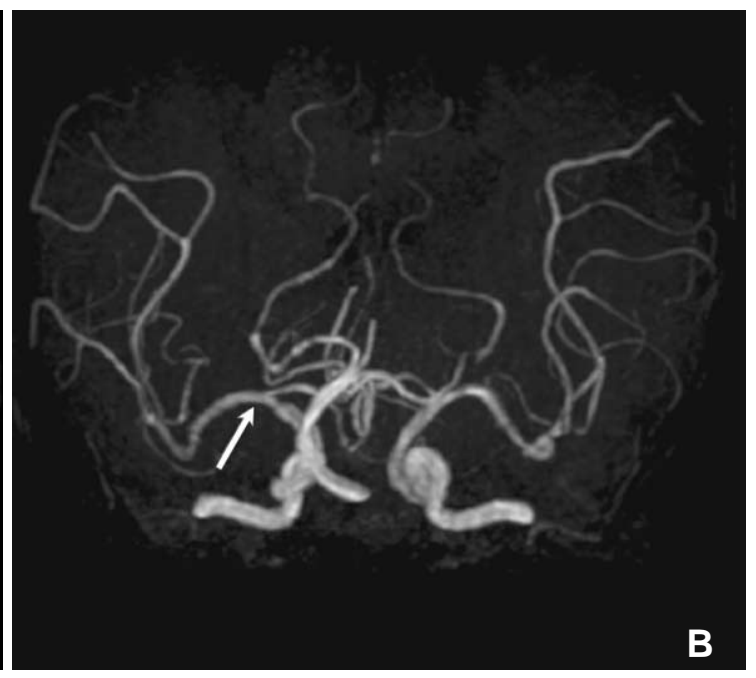

Fig. 7. A. Diffusion weighted MRI showed lacunar infarction at right corona radiate, $\mathbf{B}$. but MRA cannot show any stenosis at ipsilateral MCA (arrow). C. Axial plane of HR-MRI of MCA shows thick plaque (arrow) at right MCA. 
ing artery, the flow divider. However, superior and dorsal wall plaques near the perforating orifice have a higher prevalence in symptomatic MCA stenosis than asymptomatic MCA stenosis. Therefore, plaques near the perforating orifice may result in perforating artery territorial infarction, with or without thrombosis formation in situ [30]. These results also suggest that branch atheromatous plaque may be another cause of lacunar infarctions, in addition to pathology at the terminal arteriole.

\section{Subclinical diagnosis}

Before ICAD suddenly become symptomatic, atherosclerotic lesions progress subclinically over years. Therefore, early detection of ICAD may allow therapeutic intervention while the disease is still asymptomatic. Clinically, atherosclerosis with a low degree of stenosis still has an enhanced risk of becoming symptomatic. However, luminal imaging tools fall short in characterizing the presence of nonstenotic atherosclerotic disease. Several previous studies comparing HR-MRI and in vivo luminal imaging in patients with ICAD found that HR-MRI was able to image a small plaque that did not yield stenosis on MRA [12, 32]; HR-MRI can provide detailed information about smaller degrees of stenosis than other imaging methods. As suggested by this work, nonstenotic ICAD may turn out to be much more common than stenotic ICAD. These results correlate well with autopsy results from patients who died because of an ischemic or hemorrhagic stroke, in whom a strikingly high prevalence of ICAD was found [4].

A case series that reviewed different HR-MRI findings in posterior circulation infarction indicates that HR-MRI successfully delineates intracranial arterial lesions, suggesting that its use will lead to a further understanding of the mechanisms involved in stroke [33]. HR-MRI is able to visualize several critical atherosclerotic plaques not visualized by conventional luminal imaging, such that plausible explanations may be discovered in patients with nonfatal stroke of unknown cause (Fig. 7).

\section{DIFFERENTIAL DIAGNOSIS}

Several characteristic patterns of HR-MRI in intracranial arterial disease have been described. These are sometimes very useful in the differential diagnosis of intracranial artery disease, including dissection, Moyamoya disease (MMD) and vasculitis [28, 34, 35].

Intracranial dissection has a similar pattern to atherosclerosis, including eccentric wall thickening with enhancement. Distinguishing features in dissection are the presence of a $\mathrm{T} 1$ hyperintense intramural hematoma in the arterial wall indicating methemoglobin, a dissecting flap with a pseudolumen. Fig. 8 is an example of vertebral artery dissection. A 42-year-old male was admitted with severe headaches and was found to have eccentric stenosis at the right distal V4 on angiogram. T1 weighted HR-MRI shows intramural hematoma with high signal intensity at the stenotic segment.
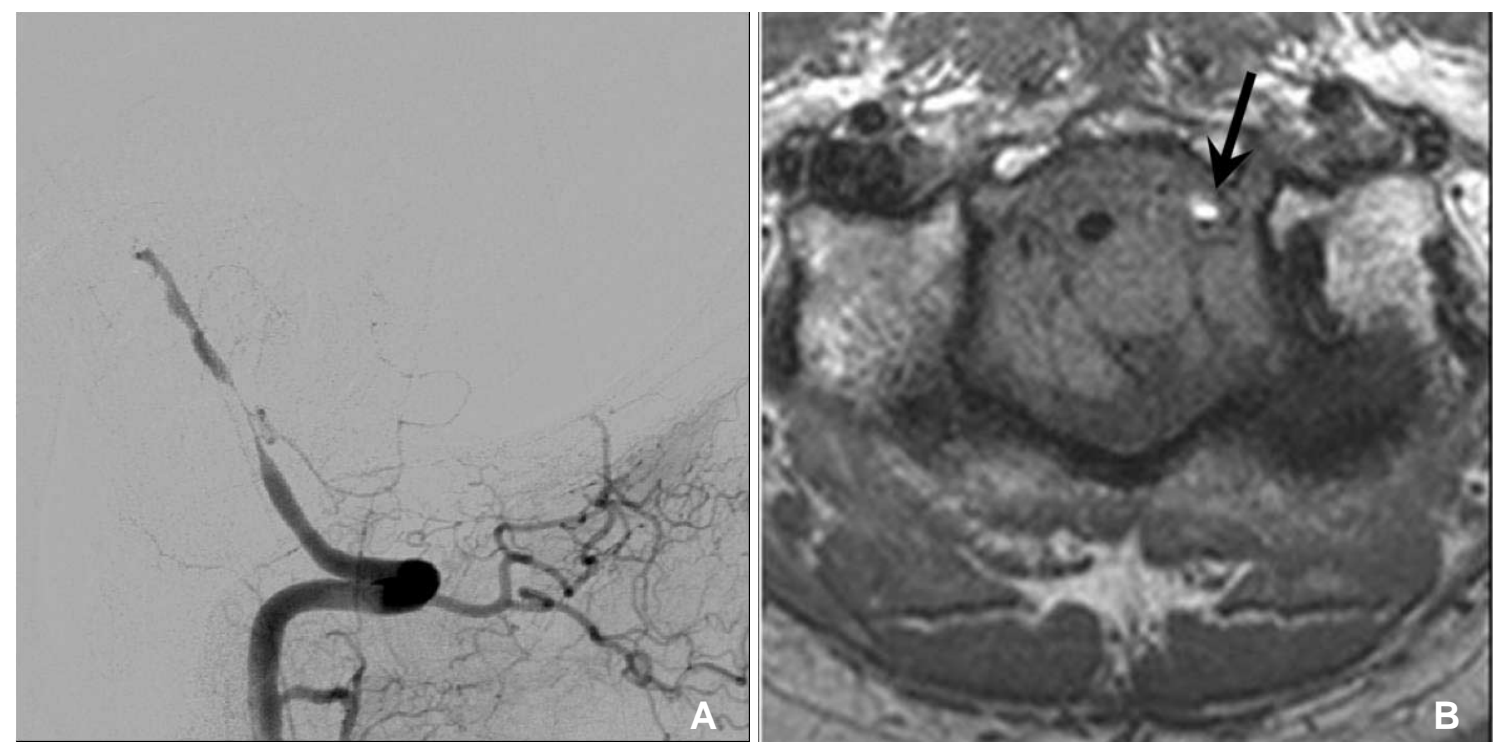

Fig. 8. In dissection, HR-MRI can reveal methemoglobin in arterial wall that show high signal intensity on T1 weighted sequence. 47 year old man with cerebellar infarction was diagnosed vertebral artery dissection. A. Vertebral angiogram shows the eccentric stenosis at distal V4. B. HR-MRI shows intramural hematoma (arrow) with high signal intensity at the stenotic segment. 
Moyamoya disease is a common cause of MCA occlusion in Asians and MMD cannot be easily distinguished from ICAD in patients having atherosclerotic risk factors, although they have differing vascular wall pathologies. On HR-MRI, MMD typically shows a constrictive pattern at the stenoocclusive segment and this finding correlates well with pathologic findings (Fig. 9).

In several previous studies of MMD disease involving HR-MRI, severely narrowed or occluded MCA branches and extensive collateralization have been described, but not wall thickening or enhancement [28, 34]. The stenotic MCAs in ICAD patients mainly showed eccentric vessel wall involvement and a heterogeneous signal intensity, whereas the MMD cases typically showed concentric vessel wall involvement and relatively homogeneous signal intensities.

Direct imaging of a vessel wall by HR-MRI may provide a confirmative diagnosis of congenital VA

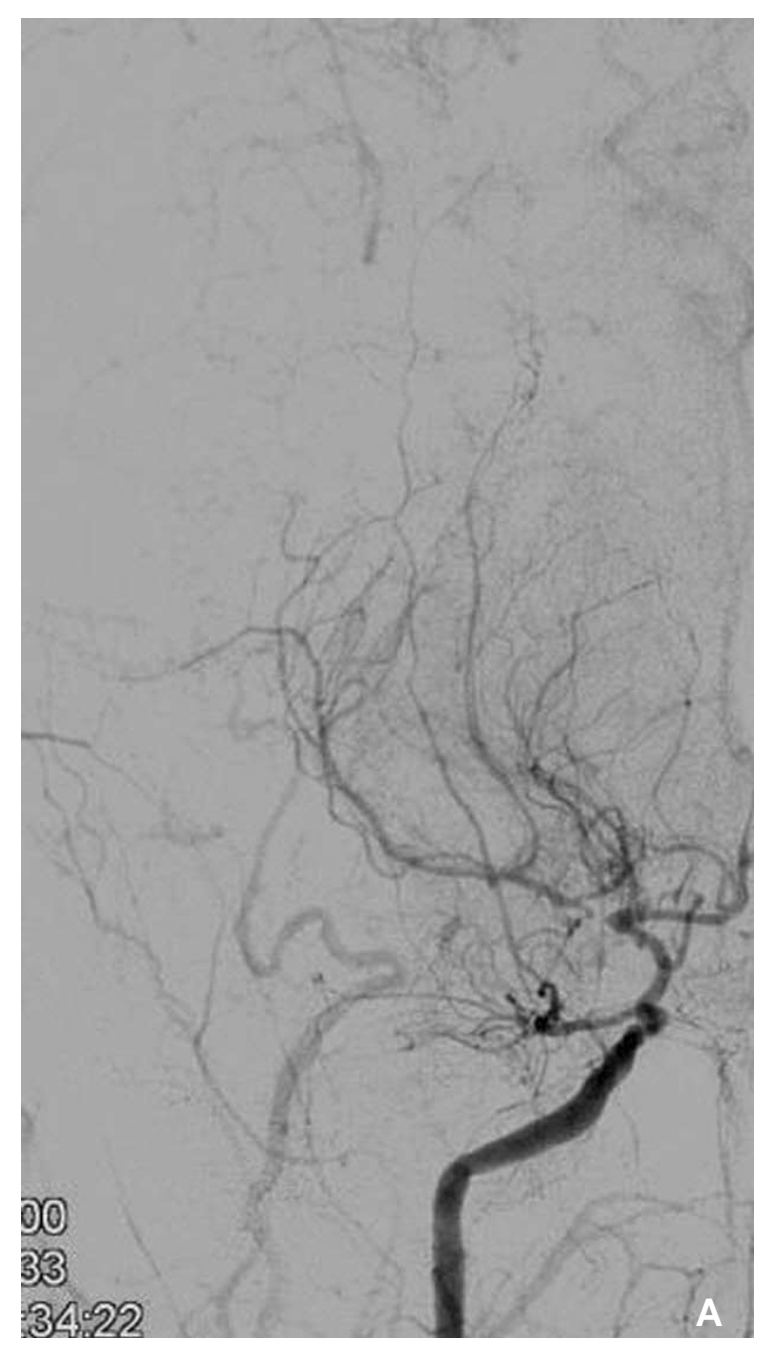

Fig. 9. Right internal carotid angiography shows severe stenosis at right carotid terminum and M1 (A). HR-MRI reveals that multiple small collateral vessels without significant MCA trunk $(\mathbf{B})$. It is a typical finding of moyamoya disease. hypoplasia and remove the need for an invasive procedure. Congenital agenesis and web-like collaterals at the MCA may be diagnosed incidentally or as a complication of intracerebral hemorrhage from an aneurysm of the collateral arteries. Because HR-MRI can visualize the occluded segment that cannot be shown on luminal imaging, it is useful to differentiate between congenital agenesis and ICAD. Occluded MCA in ICAD results in a preserved outer diameter, but congenital agenesis of the MCA shows tiny collaterals within the Sylvian fissure without a significant MCA trunk [35].

\section{PERSPECTIVE}

Recently, research on visualization of the intracranial artery using a 7.0 tesla MRI machine has been assayed. 7T MRI is able to achieve high in-plane spatial resolution and increases the signal-to-noise ratio [36, 37]. In contrast to 3T MRI, 7T MRI can demonstrate a normal intracranial vascular wall as well as pathologic segments in ICAD, allowing the earliest detection of atherosclerosis.

In the future, vessel wall imaging using multisequence MRI at 7T may be able to grade ICAD from the initial lesion to a complicated plaque with fibrous cap disruption in the same way that MRI can help assess carotid artery plaques.

We suspect that HR-MRI may play an important role in serial monitoring of ICAD due to its high repro-

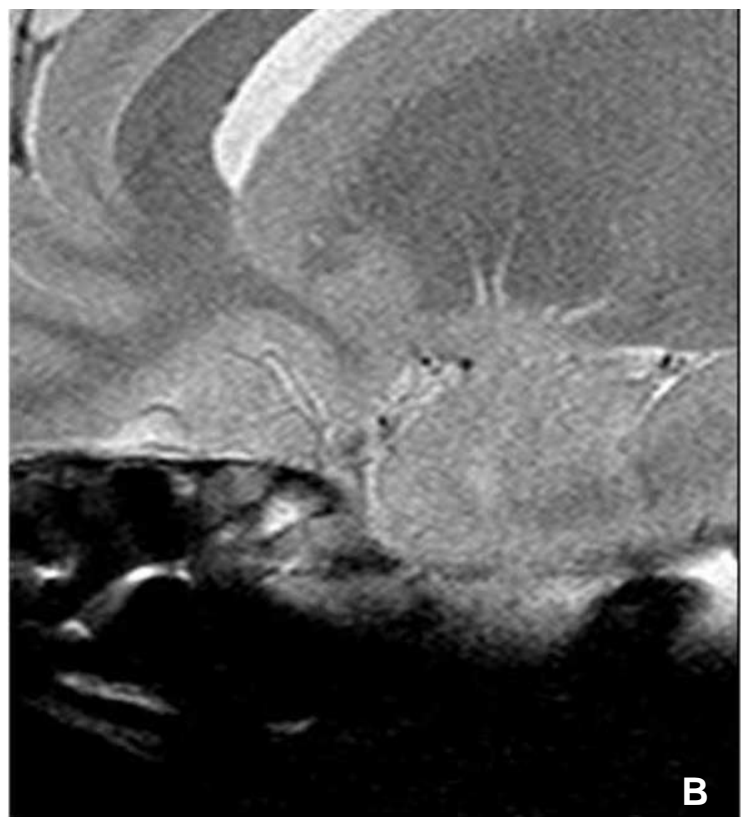


ducibility. HR-MRI helps to measure plaque volume subjectively, making HR-MRI useful in serial follow-up of ICAD, as well in assessment of response to drug therapy.

\section{CONCLUSION}

In this review, we demonstrate that HR-MRI of ICAD can disclose asymptomatic intracranial atherosclerotic plaque burden and plaque vulnerability and may provide a more in-depth understanding of ICAD. However, several limitations of ICAD studies to date are that HR-MRI is difficult to correlate with pathology, its use involves practical difficulties, and no obvious clinical gain is obtained from the use of HRMRI therapeutically. In spite of these limitations, HRMRI in ICAD appears to be of promising clinical usefulness, and interest in this modality is increasing in the fields of neurology and neuroscience. HR-MRI may help determine treatment options in ICAD and present more precise information about future risk related to the ICAD. Therefore, neurointerventionists should pay attention to the clinical usefulness of HRMRI in ICAD. In conclusion, HR-MRI for intracranial vessels can be used for in vivo analysis of plaque vulnerability, detection of subclinical ICAD, and differentiation of intracranial stenoocclusive disease.

\section{References}

1. De Silva DA, Woon FP, Lee MP, Chen CP, Chang HM, Wong $\mathrm{MC}$. South Asian patients with ischemic stroke: intracranial large arteries are the predominant site of disease. Stroke 2007;38:25922594

2. Chen PH, Gao S, Wang YJ, Xu AD, Li YS, Wang D. Classifying Ischemic Stroke, from TOAST to CISS. CNS Neurosci Ther 2012;18:452-456

3. Gorelick PB, Wong KS, Bae HJ, Pandey DK. Large artery intracranial occlusive disease: a large worldwide burden but a relatively neglected frontier. Stroke 2008;39:2396-2399

4. Mazighi M, Labreuche J, Gongora-Rivera F, Duyckaerts C, Hauw JJ, Amarenco P. Autopsy prevalence of intracranial atherosclerosis in patients with fatal stroke. Stroke 2008;39:1142-1147

5. Chung GH, Kwak HS, Hwang SB, Jin GY. High resolution MR imaging in patients with symptomatic middle cerebral artery stenosis. Eur J Radiol 2012;81:4069-4074

6. Ryu CW, Jahng GH, Kim EJ, Choi WS, Yang DM. High resolution wall and lumen MRI of the middle cerebral arteries at 3 tesla. Cerebrovasc Dis 2009;27:433-442

7. Abou-Chebl A, Steinmetz H. Critique of "Stenting versus aggressive medical therapy for intracranial arterial stenosis" by Chimowitz et al in the new England Journal of Medicine. Stroke 2012;43:616-620

8. Marks MP. Is there a future for endovascular treatment of intracranial atherosclerotic disease after Stenting and Aggressive Medical
Management for Preventing Recurrent Stroke and Intracranial Stenosis (SAMMPRIS)? Stroke 2012;43:580-584

9. Zhu XJ, Du B, Lou X, Hui FK, Ma L, Zheng BW, et al. Morphologic characteristics of atherosclerotic middle cerebral arteries on 3T high-resolution MRI. AJNR Am J Neuroradiol 2013;34:1717-1722

10. Vakil P, Vranic J, Hurley MC, Bernstein RA, Korutz AW, Habib A, et al. T1 gadolinium enhancement of intracranial atherosclerotic plaques associated with symptomatic ischemic presentations. AJNR Am J Neuroradiol 2013;34:2252-2258

11. Skarpathiotakis M, Mandell DM, Swartz RH, Tomlinson G, Mikulis DJ. Intracranial atherosclerotic plaque enhancement in patients with ischemic stroke. AJNR Am J Neuroradiol 2013;34:299-304

12. Kim YS, Lim SH, Oh KW, Kim JY, Koh SH, Kim J, et al. The advantage of high-resolution MRI in evaluating basilar plaques: a comparison study with MRA. Atherosclerosis 2012;224:411-416

13. Nishioka T, Luo H, Eigler NL, Berglund H, Kim CJ, Siegel RJ. Contribution of inadequate compensatory enlargement to development of human coronary artery stenosis: an in vivo intravascular ultrasound study. J Am Coll Cardiol 1996;27:1571-1576

14. Pasterkamp G, Wensing PJ, Post MJ, Hillen B, Mali WP, Borst C. Paradoxical arterial wall shrinkage may contribute to luminal narrowing of human atherosclerotic femoral arteries. Circulation 1995;91:1444-1449

15. Schoenhagen P, Ziada KM, Kapadia SR, Crowe TD, Nissen SE, Tuzcu EM. Extent and direction of arterial remodeling in stable versus unstable coronary syndromes : an intravascular ultrasound study. Circulation 2000;101:598-603

16. Xu WH, Li ML, Gao S, Ni J, Zhou LX, Yao M, et al. In vivo high-resolution MR imaging of symptomatic and asymptomatic middle cerebral artery atherosclerotic stenosis. Atherosclerosis 2010;212:507-511

17. Yang WQ, Huang B, Liu XT, Liu HJ, Li PJ, Zhu WZ. Reproducibility of high-resolution MRI for the middle cerebral artery plaque at 3T. Eur J Radiol 2014;83(1):e49-55

18. Saam T, Hetterich H, Hoffmann V, Yuan C, Dichgans M, Poppert $\mathrm{H}$, et al. Meta-analysis and systematic review of the predictive value of carotid plaque hemorrhage on cerebrovascular events by magnetic resonance imaging. J Am Coll Cardiol 2013;62:10811091

19. Gupta A, Baradaran H, Schweitzer AD, Kamel H, Pandya A, Delgado D, et al. Carotid plaque MRI and stroke risk: a systematic review and meta-analysis. Stroke 2013;44:3071-3077

20. Xu WH, Li ML, Gao S, Ni J, Yao M, Zhou LX, et al. Middle cerebral artery intraplaque hemorrhage: prevalence and clinical relevance. Ann Neurol 2012;71:195-198

21. Kim JM, Jung KH, Sohn CH, Moon J, Han MH, Roh JK. Middle cerebral artery plaque and prediction of the infarction pattern. Arch Neurol 2012;69:1470-1475

22. O'Brien KD, Allen MD, McDonald TO, Chait A, Harlan JM, Fishbein D, et al. Vascular cell adhesion molecule-1 is expressed in human coronary atherosclerotic plaques. Implications for the mode of progression of advanced coronary atherosclerosis. J Clin Invest 1993;92:945-951

23. O’Brien KD, McDonald TO, Chait A, Allen MD, Alpers CE. Neovascular expression of E-selectin, intercellular adhesion molecule-1, and vascular cell adhesion molecule-1 in human atherosclerosis and their relation to intimal leukocyte content. 


\section{Chang-Woo Ryu, et al.}

Circulation 1996;93:672-682

24. Moulton KS, Vakili K, Zurakowski D, Soliman M, Butterfield C, Sylvin E, et al. Inhibition of plaque neovascularization reduces macrophage accumulation and progression of advanced atherosclerosis. Proc Natl Acad Sci U S A 2003;100:4736-4741

25. Celletti FL, Waugh JM, Amabile PG, Brendolan A, Hilfiker PR, Dake MD. Vascular endothelial growth factor enhances atherosclerotic plaque progression. Nat Med 2001;7:425-429

26. Millon A, Mathevet JL, Boussel L, Faries PL, Fayad ZA, Douek PC, et al. High-resolution magnetic resonance imaging of carotid atherosclerosis identifies vulnerable carotid plaques. J Vasc Surg 2013;57:1046-1051 e1042

27. Millon A, Boussel L, Brevet M, Mathevet JL, Canet-Soulas E, Mory $\mathrm{C}$, et al. Clinical and histological significance of gadolinium enhancement in carotid atherosclerotic plaque. Stroke; a journal of cerebral circulation 2012;43:3023-3028

28. Swartz RH, Bhuta SS, Farb RI, Agid R, Willinsky RA, Terbrugge $\mathrm{KG}$, et al. Intracranial arterial wall imaging using high-resolution 3-tesla contrast-enhanced MRI. Neurology 2009;72:627-634

29. Klein IF, Lavallee PC, Mazighi M, Schouman-Claeys E, Labreuche J, Amarenco P. Basilar artery atherosclerotic plaques in paramedian and lacunar pontine infarctions: a high-resolution MRI study. Stroke 2010;41:1405-1409

30. Xu WH, Li ML, Gao S, Ni J, Zhou LX, Yao M, et al. Plaque distribution of stenotic middle cerebral artery and its clinical relevance. Stroke 2011;42:2957-2959

31. Huang B, Yang WQ, Liu XT, Liu HJ, Li PJ, Lu HK. Basilar artery atherosclerotic plaques distribution in symptomatic patients: a 3.0T high-resolution MRI study. Eur J Radiol 2013;82:e199-203

32. Li ML, Xu WH, Song L, Feng F, You H, Ni J, et al. Atherosclerosis of middle cerebral artery: evaluation with highresolution MR imaging at 3T. Atherosclerosis 2009;204:447-452

33. Chung JW, Kim BJ, Choi BS, Sohn CH, Bae HJ, Yoon BW, et al. High-Resolution Magnetic Resonance Imaging Reveals Hidden Etiologies of Symptomatic Vertebral Arterial Lesions. J Stroke Cerebrovasc Dis 2014;23:293-302

34. Kim YJ, Lee DH, Kwon JY, Kang DW, Suh DC, Kim JS, et al. High resolution MRI difference between moyamoya disease and intracranial atherosclerosis. Eur J Neurol 2013;20:1311-1318

35. Mi Kim S, Ryu CW, Jahng GH, Jong Kim E, Suk Choi W. Two Different Morphologies of Chronic Unilateral Middle Cerebral Artery Occlusion: Evaluation using High-Resolution MRI. $J$ Neuroimaging 2013 Jan 16. [Epub ahead of Print]

36. van der Kolk AG, Hendrikse J, Brundel M, Biessels GJ, Smit EJ, Visser F, et al. Multi-sequence whole-brain intracranial vessel wall imaging at 7.0 tesla. Eur Radiol 2013;23:2996-3004

37. van der Kolk AG, Zwanenburg JJ, Brundel M, Biessels GJ, Visser F, Luijten PR, et al. Intracranial vessel wall imaging at 7.0-T MRI. Stroke 2011;42:2478-2484 